\title{
Numerical Analysis of the Influence of Stent Parameters on the Fatigue Properties
}

\author{
Lin Chen, Shen Jingfeng \& Chen Bing \\ School of mechanical engineering, University of Shanghai for Science \& Technology, Shanghai, China \\ E-mail: linchen723@126.com,sh_jf@163.com, chenbing25@gmail.com
}

\author{
Xie Zhiyong \\ Microport Corporation, Shanghai, China \\ E-mail: zyxie@microport.com
}

Received: February 15, 2012

Accepted: March 6, 2012 Published: April 1, 2012

doi:10.5539/mas.v6n4p23

URL: http://dx.doi.org/10.5539/mas.v6n4p23

The research is financed by Supported by Shanghai Leading Academic Discipline Project (J50503)

\begin{abstract}
Vascular stents are small cylindrical structures implanted in stenosed vessel to support vessel wall and restore blood flow. Cycle fatigue properties of Nitinol stents is an essential feature. Structural parameters make great impact on cycle fatigue properties of Nitinol- based vascular stents. The primary aim of the paper was to investigate the effects of varying parameters of "struct V" on the pulsatile fatigue by using the finite element method (FEM). The finite element method model to analyse the fatigue properties of stent was dependant on different length, width or thickness. The results show that increasing the length, the width and the thickness could reduce the possibility of fatigue, but beyond a certain limit may play an opposite role. In conclusion, FEM is necessary tools in designing stents.
\end{abstract}

Keywords: Nitinol, Stents, Finite element method, Fatigue

\section{Introduction}

Nitinol vascular stents have been increasingly used over many years to treat vascular disease as an intravascular intervention tool, it can reduce the level of arteries injury, significantly improve restenosis within arteries and restore the smooth flow of blood. But many clinical studies show that Nitinol stent fracture occasionally occur, the main reason of fracture is periodic cyclic deformation. Due to cardiac cycle, the stented arteries do pulsatile motion. According to the 2005 FDA Guidance Document for Intravascular Stents, the finite element method is a necessary tool to analyze the fatigue and durability of intravascular stents (FDA, 2005; ASTM, 2006a). Pelton and other researchers have done many studies of diamond-shaped Nitinol stent fatigue, they found that implanted diamond-shaped Nitinol stents will be able to meet 10 years life in body as long as the amplitude of maximum principal strain is less than 0.4\% (Pelton A. R., Schroeder V. \& Mitchell M. R., 2008; Duerig T., et al., 2000; Wick A., et al., 2006; Pelton A. R., 2011). Although the assessment of pulsatile fatigue properties of the Nitinol stents is not yet mature because of the complex mechanical properties of Nitinol, designers can decrease the amplitude of the maximum principal strain to reduce the fatigue possibility of stents. In all of the structural parameters, the length, width and thickness of "V strut" play a major role to effect the strain of implanted stents. Nitinol stents were crimped and then deployed into artery, this paper compares the simulation results by varying these parameters to conclude the relationship between the structural parameters and pulsatile fatigue of stents.

Because of the complex relationship between radial force and stent failure, the association between radial force and these structural parameters has also been investigated in this paper. The finite element method (FEM) provides an excellent means to simulate the complex mechanical environment between stents and artery, including simulation highly nonlinear stress-strain relations of Nitinol stents and artery materials(Gong X. Y., et al., 2004; Auricchio F., et al., 2011; Auricchio F., et al., 2001; Prendergast P. J., et al., 2003; Migliavacca F., et al., 2005). The purpose of this simulation was to use the finite element method to evaluate the impact of varying "V strut" parameters on the pulsatile fatigue of stents. 


\section{Methods}

To complete the numerical analysis, the finite element method requires a lot of information, including the geometry and the material properties of the stents, the catheter and the artery, and the appropriate application of boundary conditions and loading, as described below.

\subsection{Model Geometry}

In each simulation model contains three parts: the stent, the artery and the catheter. The self-expanding stents were manufactured from Nitinol thin-wall tubes, the tubes were laser-cut and subsequently expanded and thermally shape-set into their final dimensions, the diameter of the final expanded stents is $8 \mathrm{~mm}$. Stents is made of two components: links and support rings, every support ring consisting of fifteen repetitive "strut V", see Figure 1.

The parameters of "strut V" are the main factors impacting on the stent fatigue (Duerig T., Pelton A. R. \& Stockel, D., 1999). The parameters of "strut V" showed in Table 2. For the aim of investigation, the stent models were labeled according to the parameters. Names were composed based on the strut length, width and thickness. The width and thickness value of $\mathrm{ABB}, \mathrm{BBB}$, and $\mathrm{CBB}$ stents are equal, but the length varying from $1.7 \mathrm{~mm}, 1.9$ $\mathrm{mm}$, to $2.1 \mathrm{~mm}$. The length and thickness of $\mathrm{BAB}, \mathrm{BBB}$, and $\mathrm{BCB}$ stents are equal, but the width varying from $0.12 \mathrm{~mm}, 0.135 \mathrm{~mm}$, and $0.15 \mathrm{~mm}$. The length and width value of BBA, BBB, and BBC stents are equal, but the thickness varying from $0.155 \mathrm{~mm}, 0.175 \mathrm{~mm}$, and $0.195 \mathrm{~mm}$. The structure of the stents is axial repeat, thus a simplified model to take one of two rings, see Figure 1. The element type of stent is C3D8I, the mesh density of width and thickness is four. The artery model is simplified to an equal thickness of cylinder, the axial length of artery model equal to stent model. In general, the thickness of equal to one-tenth of the diameter of artery, the diameter is $6 \mathrm{~mm}$, the thickness is $0.6 \mathrm{~mm}$. the element type of artery is C3D8H, the element type can accurately simulate artery which is incompressible material. The element type of catheter is SFM3D4R. The commercial FEA package ABAQUS/Standard version 6.10-1 was used in combination with the user-defined material subroutine (UMAT) for these computational analyses.

\subsection{Materials}

The vessel is modeled as an isotropic hyperelastic material as proposed by Lally et al. (2005). The model of the arterial walls was modeled using third-order Mooney-Rivlin hyperelastic constitutive equation. The general polynomial form of the strain energy density function in terms of the strain invariants is:

$$
W=a_{10}\left(I_{1}-3\right)+a_{01}\left(I_{2}-3\right)+a_{20}\left(I_{1}-3\right)^{2}+a_{11}\left(I_{1}-3\right)\left(I_{2}-3\right)+a_{30}\left(I_{1}-3\right)^{3}
$$

Table 1 summarizes the constants defining $\mathrm{W}$. The behavior of Nitinol is extremely complex, as can be seen from its uniaxial behavior shown in Figure 2. The material data required to calibrate the Abaqus material model can be obtained from the uniaxial behavior in terms of loading, unloading, reverse loading, and temperature effects. Basic material constant of Nitinol stents and artery was shown in Table 1.

\subsection{Boundary Condition}

First of all, the global coordinate systems should be converted from Cartesian coordinates to cylindrical coordinates. The simulation consists of three steps, see Figure 3:

1) Stent crimping: stents is gradually crimped to the outer diameter of $1 \mathrm{~mm}$ and leading to the stent deformation, the contact between the stent and vessel is deactivated in this step $(a \rightarrow b)$.

2) Stent deployment: from crimped configuration, the catheter is re-enlarged and consequently the stent expands against the vessel wall; the contact between the stent and the vessel is activated in this step $(b \rightarrow c)$.

3) Stent pulsatile motion: exert the pressure of $100 \mathrm{mmHg}$ on the inner surface of vessel $(\mathrm{c} \rightarrow \mathrm{d})$.

On two end surfaces of the stent, all degrees of freedom of all nodes are linear constrains with keyword EQUATION. All nodes of vessel ends and any node on end cross-section of each link component were constrained in circumferential and radial degrees of freedom.

\section{Results}

When the blood vessel and stents are in the balance, the radius of stents can be a direct reflect on the capacity of radial resistive. Finite element analysis results shows: When ABB, BBB, and CBB stents balanced with vessel, the radius gradually decreased from $3.71 \mathrm{~mm}, 3.66 \mathrm{~mm}$, to $3.63 \mathrm{~mm}$, it represents the increase in the length of "strut V" will reduce the performance of radial resistive. 
When $\mathrm{BAB}, \mathrm{BBB}$, and $\mathrm{BCB}$ stents balanced with vessel, the radius gradually decreased from $3.63 \mathrm{~mm}, 3.66 \mathrm{~mm}$, to $3.7 \mathrm{~mm}$, it represents the increase in the width of "strut V", which will enhance the performance of radial resistive. When BBA, BBB, and BBC stents balanced with vessel, the radius gradually decreased from $3.64 \mathrm{~mm}$, $3.66 \mathrm{~mm}$, to $3.68 \mathrm{~mm}$, it represents the increase in the thickness of "strut V" will enhance the performance of radial resistive.

Exerting pulse pressure on the inner surface of vessel, the amplitude of maximum principal strain of stents reflects on the fatigue property. The result shows: As the length of the "strut v" increased from $1.7 \mathrm{~mm}(\mathrm{ABB})$, $1.9 \mathrm{~mm}(\mathrm{BBB})$, to $2.1 \mathrm{~mm}(\mathrm{CBB})$, the amplitude of the maximum principal strain of stents decreased from $0.22 \%, 0.2 \%$, to $0.195 \%$. As the width of the "strut v" increased from $0.12 \mathrm{~mm}(\mathrm{BAB})$, to $0.135 \mathrm{~mm}$ (BBB), the amplitude of the maximum principal strain of stents decreased from $0.21 \%$, to $0.2 \%$, but the width increased to $0.15 \mathrm{~mm}$ (BCB), the amplitude is not decreased but increased to $0.215 \%$. As the thickness of the "strut v" increased from $0.155 \mathrm{~mm}$ (BBA), to $0.175 \mathrm{~mm}$ (BBB), the amplitude of the maximum principal strain of stents decreased from $0.22 \%$, to $0.2 \%$, but when the thickness increased to $0.195 \mathrm{~mm}(\mathrm{BBC})$, the amplitude sustained on $0.2 \%$.

\section{Discussion}

The cycle fatigue properties of Nitinol stents are important features in the consideration of stent design. Decreasing the amplitude of max principal strain can increase the fatigue-life of stents. The strain is related to the "strut V" and to the oversizing in the form (Pelton A. R., Schroeder V. \& Mitchell M. R., 2008):

$$
\varepsilon=Z \frac{\pi(\Delta D)}{M} \frac{w}{L^{2}}
$$

Where: L, and $\mathrm{w}$ are respectively the strut length and width as shown in Figure 1(a); $\mathrm{Z}$ is a function of the material non-linearity; $\Delta \mathrm{D}$ is approximately equal to the oversizing of the stent in vivo; $\mathrm{M}$ is the circumferential number of strut cells per strut row. This equation confirmed that some geometric factors impact the stent strain. The relative COF is proportional to the structural stiffness in the following form (Pelton A. R., 2011):

$$
C O F \propto \text { Stiffness } \approx \frac{t w^{3}}{L^{3}}
$$

As such, for a given design, minor adjustments to $\mathrm{w}$ and $\mathrm{L}$ can be made to fine tune the stiffness and thereby the COF. Stent designer can improve the performance of the stent support by reducing the length, increasing the width and thickness of the stent. The fatigue-life of stents is not necessarily will be enhanced and sometimes may be reduced due to support performance improvement. Based on our findings, reducing the length of the "strut V" can increase the stent support performance, but it also reduce the stent fatigue life. Increasing the width of the "strut V" will increase the performance of the stent support, can also increase the fatigue perporties, but it is limited, more than one value will have the opposite effect. Changing the thickness has the similar effect. Stent designer should consider the performance of the stent support and fatigue resistance. They can pick out the most suitable stent by analyzing different structural parameters of the "strut V".

\section{Conclusion}

The rules that the changes of "strut V" parameters impact on the capacity of radial resistive is obvious. However the rules that the changes of "strut V" parameters impact on the property of fatigue is complex, we can not simply change the length, width, or thickness of "strut V" to achieve our goal. Because of the complex relationship between radial force and the fatigue property, in other words, changes of the equilibrium position between stents and vessel will affect the deformation of the stents, and impact on fatigue-life of the stents.

\section{Refferences}

ASTM. (2006a). F2477-06, Standard test method for in vitro pulsatile durability testing of vascular stents. ASTM International.

Auricchio, F., Loreto, M. Di., \& Sacco, E. (2001). Finite-element analysis of a stenotic artery revascularization through a stent insertion. Computer Methods in Biomechanics and Biomedical Engineering, 1-15.

Auricchio, F., Cont, I. M., et al. (2011). Carotid artery stenting simulation: From patient-specific images to finite element analysis. Medical Engineering \& Physics, 33, 281-289. http://dx.doi.org/10.1016/j.medengphy.2010.10.011

Duerig, T., Pelton, A. R., \& Stockel, D. (1999). An Overview of Nitinol Medical Applications. Materials Science and Engineering, 273-275, 149-160. http://dx.doi.org/10.1016/S0921-5093(99)00294-4

Duerig, T., Tolomeo, D., et al. (2000). An overview of superelastic stent design. Min. Invas. Ther. \& Allied 
Technol, 9(3-4), 235-246. http://dx.doi.org/10.1080/13645700009169654

FDA. (2005). Guidance document for intravascular stents.

Gong, X. Y., Pelton, A. R., Duerig, T. W., et al. (2004). Finite Element Analysis and Experimental Evaluation of superelastic Nitinol Stens, Proceedings of SMST-2003, Monterey, CA(eds.).

Lally, C., Dolan, F., \& Prendergast, P. J. (2005). Cardiovascular stent design and vessel stresses: a finite element analysis. Journal of Biomechanics, 38(3), 1574-1581. http://dx.doi.org/10.1016/j.jbiomech.2004.07.022

Migliavacca, F., Schievano, S., Ricciardi, G., \& Dubini, G. (2005). Does numerical simulation reflect really the mechanical behaviour of a stent? ESVB-2005, New Technologies in Vascular Biomaterials, Fundamental About Stent.

Pelton, A. R., Schroeder, V., \& Mitchell, M. R. (2008). Fatigue and durability of Nitinol. Journal of the Mechanical Behavior of Biomedical Materials, 1(2), 153-164. http://dx.doi.org/10.1016/j.jmbbm.2007.08.001

Pelton, A. R. (2011). Nitinol Fatigue: A Review of Microstructures and Mechanisms. Journal of Materials Engineering and Performance, 2, 1059-1063.

Prendergast, P. J., Lally, C., Daly, S., Reid, A. J., \& Lee, T. C. (2003). Analysis of prolapse in cardiovascular stents: A constitutive equation for vascular tissue and finite-element modelling. Journal of Biomechanical Engineering, 125(5), 692-699. http://dx.doi.org/10.1115/1.1613674

Wick, A., Gong, X. Y., Fino, J., Sheriff, J., \& Pelton, A. R. (2006). Bending Fatigue Characteristics of Nitinol. Proceeding of SMST-2004, ed.M.Mertmann, Baden-Baden, Germany, 361-366.

Table 1. Material constants for the stent and artery model

\begin{tabular}{ccccccccc}
\hline Model & \multicolumn{7}{c}{ Material constant } \\
\hline \multirow{2}{*}{ Stent } & $E_{A}$ & $E_{M}$ & $v$ & $\varepsilon^{L}$ & $\sigma_{A M}^{s}$ & $\sigma_{A M}^{f}$ & $\sigma_{M A}^{s}$ & $\sigma_{M A}^{f}$ \\
& $40000 \mathrm{Mpa}$ & $32000 \mathrm{Mpa}$ & 0.33 & 0.045 & $400 \mathrm{Mpa}$ & $500 \mathrm{Mpa}$ & $140 \mathrm{Mpa}$ & $50 \mathrm{Mpa}$ \\
\hline \multirow{2}{*}{ Artery } & $a_{10}$ & $a_{01}$ & $a_{20}$ & $a_{11}$ & $a_{30}$ & & & \\
(Mpa) & 0.0189 & 0.00275 & 0.59043 & 0.00275 & 0 & & \\
\hline
\end{tabular}

Table 2. Geometric data and results

\begin{tabular}{lllllll}
\hline & \multicolumn{2}{l}{ Parameters of stent model } & \multicolumn{2}{l}{ Result } \\
\cline { 2 - 7 } Model & $\begin{array}{l}\text { Strut } \\
\text { length(mm) }\end{array}$ & $\begin{array}{l}\text { Strut } \\
\text { width(mm) }\end{array}$ & $\begin{array}{l}\text { Strut } \\
\text { thickness(mm) }\end{array}$ & $\begin{array}{l}\text { Amplitude of } \\
\text { maximum } \\
\text { principal } \\
\text { strain }\end{array}$ & $\begin{array}{l}\text { Maximum } \\
\text { stent } \\
\text { radius(mm) }\end{array}$ & $\begin{array}{l}\text { Radial } \\
\text { displacement } \\
\text { under pulsatile } \\
\text { pressure(mm) }\end{array}$ \\
\hline ABB & 1.7 & 0.135 & 0.175 & $0.22 \%$ & 3.71 & 0.077 \\
BBB & 1.9 & 0.135 & 0.175 & $0.20 \%$ & 3.66 & 0.081 \\
BAB & 1.9 & 0.12 & 0.175 & $0.21 \%$ & 3.63 & 0.087 \\
BCB & 1.9 & 0.15 & 0.175 & $0.22 \%$ & 3.7 & 0.075 \\
BBA & 1.9 & 0.135 & 0.155 & $0.22 \%$ & 3.64 & 0.085 \\
BBC & 1.9 & 0.135 & 0.195 & $0.20 \%$ & 3.68 & 0.078 \\
CBB & 2.1 & 0.135 & 0.175 & $0.20 \%$ & 3.63 & 0.086 \\
\hline
\end{tabular}


a

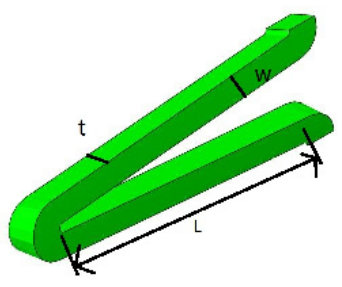

b

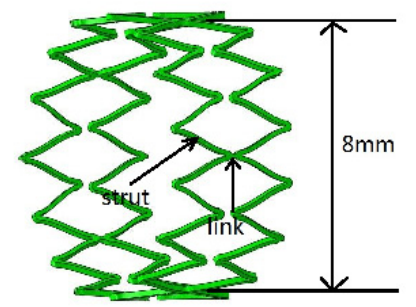

(a) "Strut V" (b) Studied part of the stent

Figure 1. Geometric model of the stent

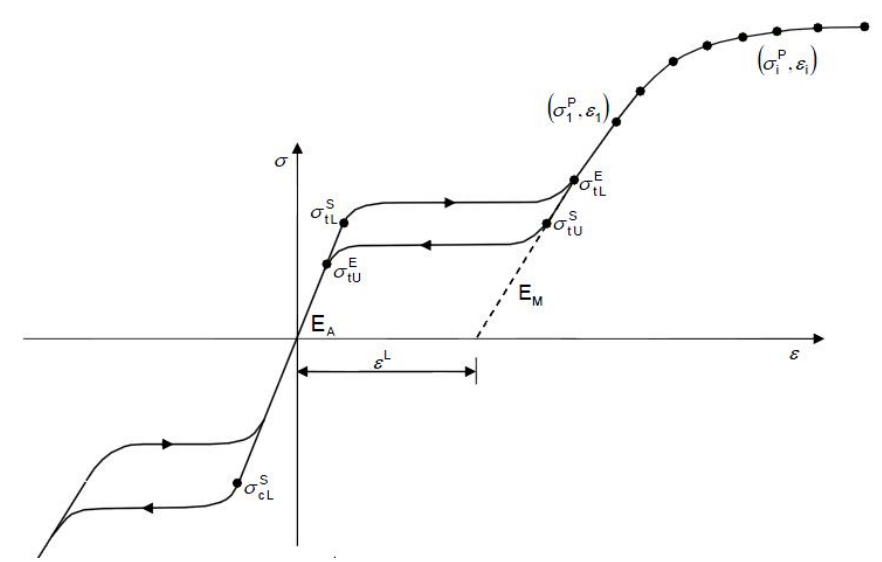

Figure 2. Stress-strain curve of Nitinol material
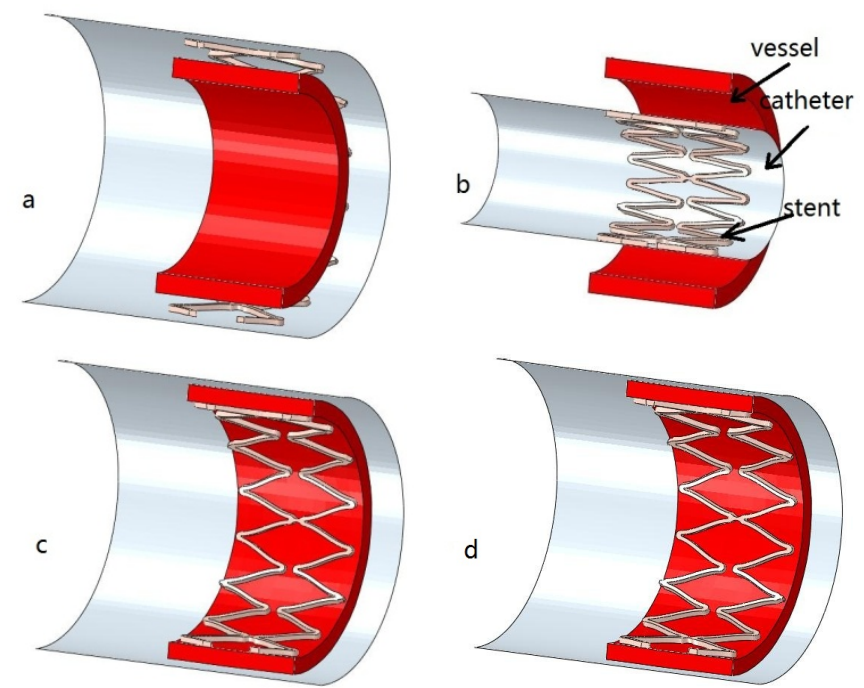

(a) starting configuration of the FE model, (b) stent crimped in the delivery system; (c) stent deployed in the vessel, (d) pulsatile pressure in the vessel

Figure 3. Simulation of stent implantation 